\title{
High expression of muscarinic acetylcholine receptor 3 predicts poor prognosis in patients with pancreatic ductal adenocarcinoma
}

This article was published in the following Dove Press journal:

OncoTargets and Therapy

31 October 2016

Number of times this article has been viewed

\author{
Lingfu Zhang' \\ Dianrong Xiu' \\ Jun Zhan ${ }^{2,3}$ \\ Xiaokun $\mathrm{He}^{3}$ \\ Limei Guo 4,5 \\ Jilian Wang' \\ Ming Tao' \\ Wei Fu' \\ Hongquan Zhang 2,3 \\ 'Department of General Surgery, \\ Peking University Third Hospital, \\ ${ }^{2}$ Key Laboratory of Carcinogenesis \\ and Translational Research, Ministry \\ of Education, State Key Laboratory \\ of Natural and Biomimetic Drugs, \\ ${ }^{3}$ Laboratory of Molecular Cell Biology \\ and Tumor Biology, Department of \\ Anatomy, Histology and Embryology, \\ ${ }^{4}$ Department of Pathology, Peking \\ University Health Science Center, \\ ${ }^{5}$ Department of Pathology, Peking \\ University Third Hospital, Beijing, \\ People's Republic of China
}

Correspondence: Dianrong Xiu Department of General Surgery, Peking University Third Hospital, \#49 Huan-yuan North Road, Beijing I00191, People's Republic of China

Tel +86 I082267320

Email xiudianrong@hotmail.com

Hongquan Zhang

Laboratory of Molecular Cell Biology and Tumor Biology, Department of Anatomy, Histology and Embryology, Peking University Health Science Center, \#38 Xue Yuan Road, Beijing 100191, People's Republic of China

Tel +86 I0 82802424

Email hongquan.zhang@bjmu.edu.cn
Aims: Recent studies showed that muscarinic acetylcholine receptor 3 (M3), as a muscarinic acetylcholine receptor family member that plays an important role in normal physiological function, is engaged in cancer progression. However, the role of M3 in pancreatic ductal adenocarcinoma (PDAC) is not known. The aim of this study is to investigate the expression and prognostic value of M3 in patients with PDAC.

Materials and methods: The localization and expression of M3 in PDAC were examined by immunohistochemistry. VAChT was employed to detect parasympathetic nerve fibers in the corresponding M3 PDAC tissues. The correlation between M3 expression and patients' survival was assessed by Kaplan-Meier analysis.

Results: M3 was discovered predominantly localized in the cell cytoplasm and expressed in all specimens of PDAC patients. Significant correlation was noted between increased M3 intensity and high grade of PDAC $(P<0.01)$, more lymph node metastasis $(P<0.01)$ as well as shorter patient overall survival $(P<0.01)$. Morphologically, cells with high M3 expression were more frequently located at the invasive tumor front/tumor budding cells, metastatic lymph nodes and parasympathetic nerve fibers.

Conclusion: High expression of M3 is a prognostic marker for PDAC.

Keywords: PDAC, muscarinic acetylcholine receptor 3, M3, tumor budding, parasympathetic nerve fiber, prognosis

\section{Introduction}

Pancreatic ductal adenocarcinoma (PDAC) is one of the most lethal cancers because of its propensity for both local invasion and distant metastasis, as well as its resistance to therapy. ${ }^{1}$ It is of great significance to explore new molecules to give a more accurate prediction and guide a more efficient treatment.

The muscarinic acetylcholine receptors (mAChRs) contain a family of five related G protein-coupled receptors (GPCRs, denoted as M1 to M5 and encoded by the genes CHRM1 to CHRM5), belonging to the $\alpha$-branch of class A GPCRs. ${ }^{2}$ The mAChRs play an important role in human physiology, such as the regulation of heart rate, smooth muscle contraction, glandular secretion and many central nervous system fundamental functions. ${ }^{3}$ Currently, drugs targeting muscarinic receptors are applied in the treatment of several pathophysiological conditions such as chronic obstructive pulmonary disease, overactive bladder and Sjögren's syndrome.,

Recent studies have demonstrated that overexpression of muscarinic acetylcholine receptor 3 (M3) has been correlated with tumor progression and metastasis in certain human colon cancer, gastric cancer and lung cancer..$^{5-7}$ However, the role of M3 in 
PDAC has not been fully investigated yet. We hypothesized that overexpression and activation of M3 may contribute to the development and metastasis of PDAC and it may be a novel therapeutic target for PDAC. Therefore, we examined the expression of M3 in PDAC tissues and explored the relationship between its expression and clinicopathological features of PDAC patients.

\section{Materials and methods}

\section{Patient tumor sections}

This study was approved by the Ethics Committee of Peking University Third Hospital, Beijing, the People's Republic of China (permit no IRB00006761-2015015) who deemed patient consent was not necessary as data were analyzed anonymously. The procedures for handling human materials were in line with the ethical standards of the 1975 Declaration of Helsinki, which was amended in 1983. Primary PDAC specimens $(n=58)$ were derived from patients who underwent pancreatectomies with clear surgical margins from the General Surgery Department of Peking University Third Hospital. Adjacent normal pancreatic tissue samples $(n=58)$ were obtained at least $2 \mathrm{~cm}$ away from tumor tissues in the same group of patients. All patients were subject to surgery from February 2007 to December 2010, and the follow-up utilized for survival analyses was ended in December 2014. The clinical staging of these patients followed the NCCN Guidelines version 2.2015 staging for pancreatic cancer (available at www.nccn.com). Patients who received chemotherapy prior to surgery were excluded in this study. The clinicopathological characteristics of all patients are shown in Table 1.

\section{Immunohistochemistry}

All tumors employed for this investigation were reviewed by at least two pathologists for final diagnosis. Archived formalin-fixed paraffin-embedded PDAC tumor tissue sections were dealt with as described previously. ${ }^{8}$ After deparaffinization in xylene, the sections were rehydrated in graded alcohols. Endogenous peroxidase was quenched with 3\% hydrogen peroxide in methanol at room temperature $\left(25^{\circ} \mathrm{C}\right)$. The sections were placed in a $95^{\circ} \mathrm{C}$ solution of $0.01 \mathrm{M}$ sodium citrate buffer ( $\mathrm{pH}$ 6.0) for antigen retrieval. A rabbit anti-M3 polyclonal antibody (1:200; Santa Cruz, Dallas, TX, USA) was used for the detection of $\mathrm{M} 3$, and a rabbit polyclonal antiVAChT antibody (1:100; ab-68984; Abcam, Cambridge, MA, USA) was employed for the detection of parasympathetic nerve fiber. The primary antibody was applied overnight at $4^{\circ} \mathrm{C}$. PV9000 two-step plus a poly-horseradish peroxidase antimouse/rabbit immunoglobulin $\mathrm{G}$ detection system (Zhongshan Jinqiao, Jiaxing, the People's Republic of China)
Table I Characteristics of clinical data of PDAC patients

\begin{tabular}{|c|c|c|}
\hline Characteristics & Number & $\%$ \\
\hline Age: median (range), years & $63.5(23,80)$ & \\
\hline \multicolumn{3}{|l|}{ Gender } \\
\hline Female & 29 & 50 \\
\hline Male & 29 & 50 \\
\hline \multicolumn{3}{|l|}{ Location of the tumor } \\
\hline Head and neck & 49 & 84.5 \\
\hline Body and tail & 9 & 15.5 \\
\hline \multicolumn{3}{|l|}{ TNM category } \\
\hline TI & 4 & 6.9 \\
\hline $\mathrm{T} 2$ & 32 & 55.2 \\
\hline $\mathrm{T} 3$ & 22 & 37.9 \\
\hline No & 28 & 48.3 \\
\hline NI & 30 & 51.7 \\
\hline Mo & 58 & 100 \\
\hline MI & 0 & 0 \\
\hline \multicolumn{3}{|l|}{ AJCC stage } \\
\hline $\mathrm{IA}$ & 2 & 3.4 \\
\hline IB & 19 & 32.8 \\
\hline IIA & 7 & 12.1 \\
\hline IIB & 30 & 51.7 \\
\hline \multicolumn{3}{|l|}{ Survival status } \\
\hline Alive & 19 & 32.8 \\
\hline Dead & 39 & 67.2 \\
\hline
\end{tabular}

Note: AJCC is a classification system developed by the American Joint Committee on Cancer for describing the extent of disease progression in cancer patients. Abbreviations: PDAC, pancreatic ductal adenocarcinoma; TNM, tumor-nodemetastasis.

was then applied. Detection was performed using the Dako Envision System (Dako, Glostrup, Denmark), followed by chromogen detection with diaminobenzidine. Hematoxylin was used for counterstaining. Negative controls were performed by omitting the use of the primary antibody.

\section{Semi-quantitative scoring}

Semi-quantitative scoring of antibody staining on the slides was examined and checked by two pathologists, and scores were made without prior knowledge of clinical information. There are no common criteria for the grading of M3 in PDAC, and hence we take our criteria according to the grading of M3 in other cancers, such as lung cancer and endometrial cancer. Staining intensity and percentage of tumor cells stained were utilized in evaluating M3 staining on the tumor tissue section $(0=$ not detected, or $<1 \%$ positive cells; $1=$ weak, or $1 \%-40 \%$ positive cells; $2=$ moderate, or $40 \%-70 \%$ positive cells and 3-strong, or $>70 \%$ positive cells). "Score 3" was defined as high expression and "scores 1 and 2" as low expression. Cases with discrepancies were reevaluated simultaneously by the two pathologists until a consensus was reached. The value of kappa is 0.763 . If there were discrepancies between staining intensity and percentage of tumor cells, the higher grade was chosen to represent the grade. 


\section{Statistical analysis}

Differences of M3 expression among various subgroups were identified through the Kruskal-Wallis rank sum test. Student's $t$-test was used for paired studies. The Kaplan-Meier method was performed to represent survival curves, and the log-rank test was applied to verify significant differences in survival time. Association of overall survival (OS) with M3 expression was examined by Cox regression model using backward stepwise (likelihood ratio) method. Results were considered statistically significant when $P<0.05$ (two-sided) using SPSS version 21.0 (SPSS Inc., Chicago, IL, USA).

\section{Results}

\section{M3 is not expressed in adjacent noncancer pancreatic tissue and increased in PDAC}

To detect the M3 expression in PDAC, we examined a cohort of 58 patients with an age distributed from 23 to 80 years. We examined M3 expression in noncancer pancreatic tissues and found that M3 was frequently absent in these tissues (Figure 1A). But M3 expression was observed in all cancer tissues, which was expressed predominantly in the cytoplasm of PDAC cells. Cytoplasmic M3 staining on tumor tissue slides was assessed using the following criteria: grade $1=$ weak (Figure 1B), grade $2=$ moderate (Figure 1C) and grade $3=$ high (Figure 1D). The results showed that low
M3 (1-2) expression and high M3 expression accounted for $41.4 \%$ (24) and $58.6 \%$ (34), respectively. However, only low expressions of M3 were detected in 21 (36.2\%) cases of paired adjacent noncancer tissues and the remaining 37 (63.8\%) showed no expression of M3. In addition, we compared the expression of M3 protein in PDAC tissues and the corresponding adjacent noncancer tissues; M3 expression in PDAC was also found to be increased significantly $(P=0.001)$ (Figure 1E). Taken together, these findings indicated that M3 expression is enhanced in PDAC patients and suggested that M3 may be involved in the progression of PDACs.

\section{M3 expression was associated with clinicopathological variables in PDAC}

Associations between M3 expression levels and clinicopathological variables are summarized in Table 2. The most obvious correlation was identified between $\mathrm{M} 3$ expression and PDAC N stage, in which the expression level of M3 was increased from N0 to N1 $(P=0.019)$. Similarly, the higher American Joint Committee on Cancer (AJCC) stage correlated with higher M3 expression $(P=0.032)$. However, M3 expression was not found to be related significantly with patients' age $(P=0.329)$, gender $(P=1.000)$, location of the tumor $(P=0.869)$, T stage $(P=0.409)$, $\mathrm{G}$ grade $(P=0.843)$, vessel invasion $(P=0.264)$ and neural invasion $(P=0.684)$.
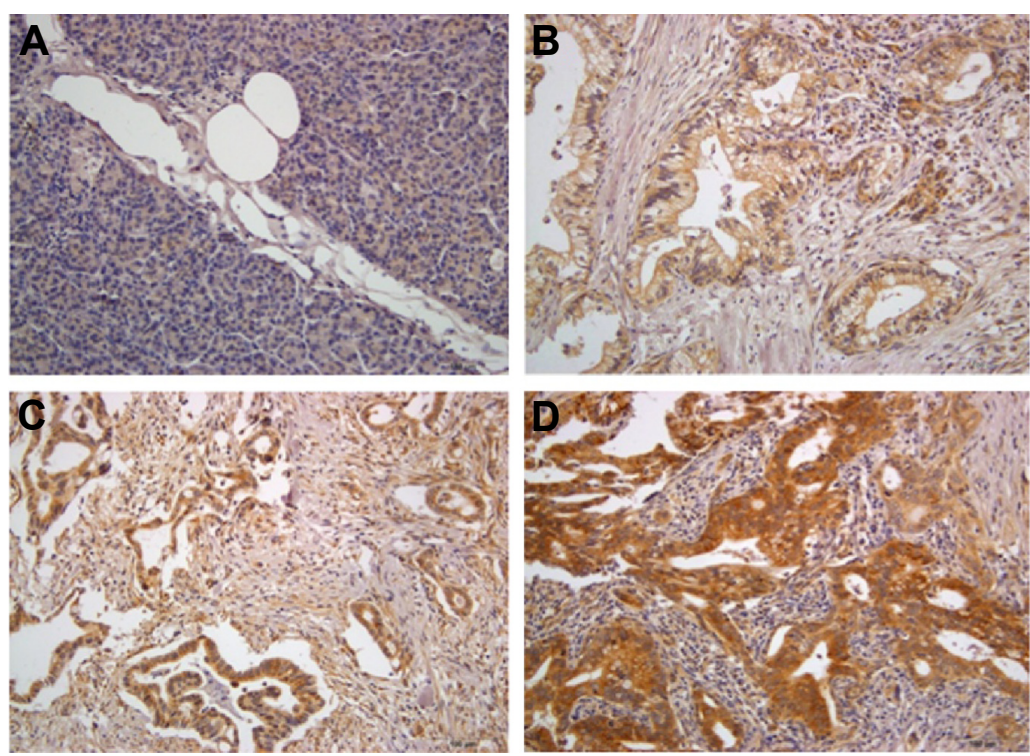

E

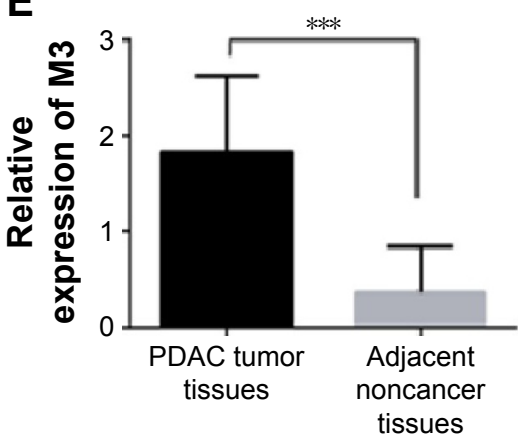

Figure I M3 is not expressed in adjacent noncancer pancreatic tissue but expressed in PDAC.

Notes: IHC staining was performed to examine the expression of M3 in adjacent noncancer pancreatic and PDAC tissues. The representative photographs are shown. (A) No expression of M3; (B) weak expression of M3; (C) moderate expression of M3; (D) high expression of M3; (E) M3 expression between PDAC tissues and corresponding adjacent noncancer pancreatic tissues. $* * * P=0.00 \mathrm{I}$. Magnification $\times 200$.

Abbreviations: M3, muscarinic acetylcholine receptor 3; PDAC, pancreatic ductal adenocarcinoma; IHC, immunohistochemical. 
Table 2 Correlation of M3 expression with clinicopathological features in PDAC

\begin{tabular}{|c|c|c|c|}
\hline Characteristics & $\begin{array}{l}\text { Low M3 } \\
\text { expression } \\
\text { group }(n=24)\end{array}$ & $\begin{array}{l}\text { High } M 3 \\
\text { expression } \\
\text { group }(n=34)\end{array}$ & $P$-value \\
\hline Age & $58.4 \pm 10.4$ & $62.9 \pm 13.9$ & 0.329 \\
\hline Gender & & & 1.000 \\
\hline Female & 12 & 17 & \\
\hline Male & 12 & 17 & \\
\hline Location of the tumor & & & 0.869 \\
\hline Head and neck & 21 & 28 & \\
\hline Body and tail & 3 & 6 & \\
\hline T stage & & & 0.409 \\
\hline 1 & 3 & 1 & \\
\hline 2 & 13 & 19 & \\
\hline 3 & 8 & 14 & \\
\hline $\mathrm{N}$ stage & & & $0.019 *$ \\
\hline 0 & 16 & 12 & \\
\hline 1 & 8 & 22 & \\
\hline AJCC stage & & & $0.032^{*}$ \\
\hline IA & 2 & 0 & \\
\hline IB & 9 & 10 & \\
\hline$\| \mathrm{A}$ & 5 & 2 & \\
\hline IIB & 8 & 22 & \\
\hline G grade & & & 0.843 \\
\hline 1 & 2 & 1 & \\
\hline 2 & 18 & 26 & \\
\hline 3 & 3 & 6 & \\
\hline 4 & I & 1 & \\
\hline Vessel invasion & & & 0.264 \\
\hline Positive & 4 & 10 & \\
\hline Negative & 20 & 24 & \\
\hline Neural invasion & & & 0.684 \\
\hline Positive & 10 & 16 & \\
\hline Negative & 14 & 18 & \\
\hline
\end{tabular}

Notes: $P$-values for age were calculated by the Mann-Whitney U-test, and other $P$-values were calculated by the chi-square tests or Fisher's exact test. *Significant $P$-value, $P<0.05$. AJCC is a classification system developed by the American Joint Committee on Cancer for describing the extent of disease progression in cancer patients.

Abbreviations: M3, muscarinic acetylcholine receptor 3; PDAC, pancreatic ductal adenocarcinoma.

\section{M3 expression was of prognostic value in PDAC}

To determine if M3 expression was correlated with patient survival, we examined patients with available follow-up data of up to 84 months. We found that 39 (67.2\%) patients died during follow-up and 19 (32.8\%) patients were alive or censored. The postoperative median survival time of the follow-up patients was 15.5 months. Patients with low or high M3 expression had median survival times of 28.0 and 12.0 months, respectively. Through Kaplan-Meier analysis, we found that the survival of patients with high M3 expression was shorter than that in the low-expression groups $(P=0.003)$ (Figure 2). In addition, the advanced clinical

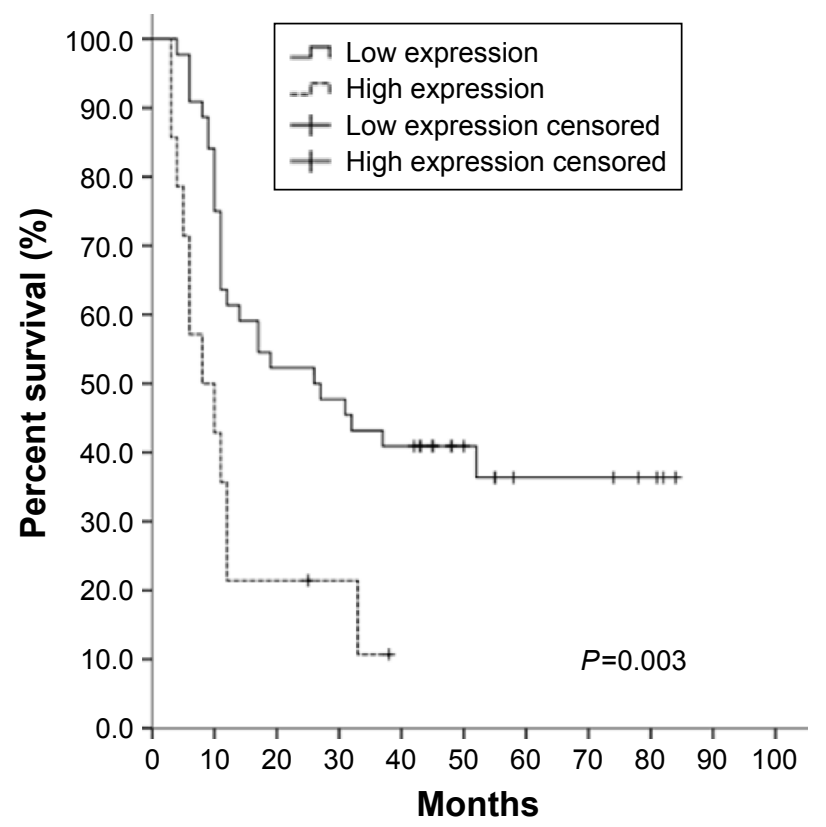

Figure 2 Increased expression of M3 predicts poor OS for PDAC patients. Notes: Two groups of PDAC patients with high and low M3 expression were examined by Kaplan-Meier analysis with a log-rank of $P=0.003$.

Abbreviations: M3, muscarinic acetylcholine receptor 3; OS, overall survival; PDAC, pancreatic ductal adenocarcinoma.

AJCC stage $(P=0.001)$, lymph node metastasis $(P<0.001)$ and neural invasion $(P=0.011)$ also predicted shorter OS for PDAC patients. In contrast, no prognostic significance was found for OS with gender $(P=0.953)$, location of the tumor ( $P=0.409)$, T stage ( $P=0.080), \mathrm{G}$ grade $(P=0.119)$ and vessel invasion $(P=0.067)$ of the patients, respectively (Table 3$)$. The multivariate Cox regression model using backward stepwise (likelihood ratio) method was employed to analyze the prognostic factors of OS in PDAC patients; in this process, T stage, N stage, AJCC stage, perineural invasion and $\mathrm{G}$ grade were included. M3 expression and AJCC stage were involved as dependent variables in the model. The result indicated that the presence of high M3 expression maintained its unfavorable impact on prognosis $(P=0.022)$. Patients with high M3 expression had a 2.270 times elevated risk of death after adjusting for the remaining features in comparison to patients with low M3 expression (Table 4). Taken together, these results indicate that $\mathrm{M} 3$ is a factor that can predict the prognosis for PDAC patients.

\section{The characteristics of $M 3$ expression in invasive tumor front (ITF)/tumor budding and metastatic lymph node}

Enhanced expression of M3 correlated with shorter patient survival in PDAC. However, the contributory role of M3 
Table 3 The effect of clinicopathological characteristics on OS by univariate Kaplan-Meier survival analysis

\begin{tabular}{|c|c|c|c|}
\hline & $\begin{array}{l}\text { Median OS } \\
\text { (months) }\end{array}$ & $\begin{array}{l}\text { Hazard } \\
\text { ratio }\end{array}$ & $P$-value \\
\hline Gender & & & 0.953 \\
\hline Female & 17.0 & 1.000 & \\
\hline Male & 14.0 & 0.982 & \\
\hline Location of the tumor & & & 0.409 \\
\hline Head and neck & 14.0 & 1.000 & \\
\hline Body and tail & 33.0 & 0.682 & \\
\hline T stage & & & 0.080 \\
\hline 1 & 26.0 & 1.000 & \\
\hline 2 & 12.0 & 0.958 & \\
\hline 3 & 11.0 & 1.925 & \\
\hline$N$ stage & & & $<0.00 I^{*}$ \\
\hline 0 & 52.0 & 1.000 & \\
\hline 1 & 10.0 & 3.557 & \\
\hline AJCC stage & & & $0.00 I^{*}$ \\
\hline IA & 19.0 & 1.000 & \\
\hline IB & - & 0.772 & \\
\hline IIA & 31.0 & 1.893 & \\
\hline IIB & 10.0 & 3.640 & \\
\hline Vessel invasion & & & 0.119 \\
\hline Positive & 10.0 & 1.000 & \\
\hline Negative & 19.0 & 1.713 & \\
\hline Neural invasion & & & $0.011 *$ \\
\hline Positive & 11.0 & 1.000 & \\
\hline Negative & 27.0 & 2.185 & \\
\hline G stage & & & 0.067 \\
\hline 1 & - & 1.000 & \\
\hline 2 & 17.0 & 2.948 & \\
\hline 3 & 10.0 & 4.997 & \\
\hline 4 & 6.0 & 11.959 & \\
\hline M3 expression & & & $0.003^{*}$ \\
\hline Low & 26.0 & 1.000 & \\
\hline High & 8.0 & 2.629 & \\
\hline
\end{tabular}

Notes: All the $P$-values were calculated by the log-rank test. *Significant $P$-value, $P<0.05$.

Abbreviations: OS, overall survival; AJCC, American Joint Committee on Cancer; M3, muscarinic acetylcholine receptor 3 .

Table 4 Summary of important risk factors for OS in multivariate Cox regression analysis

\begin{tabular}{|c|c|c|c|c|c|c|}
\hline & $\mathbf{n}$ & $\beta$ & SE & $P$ value* & HR & $95 \% \mathrm{Cl}$ \\
\hline \multicolumn{7}{|l|}{ AJCC stage } \\
\hline Stage IA & 2 & & & & 1.000 & \\
\hline Stage IB & 19 & -0.306 & 0.770 & & 0.737 & $0.163-3.333$ \\
\hline Stage IIA & 7 & -0.703 & 0.398 & & 0.495 & $0.227-1.081$ \\
\hline Stage IIB & 30 & 0.225 & 0.432 & 0.006 & 1.253 & $0.537-2.922$ \\
\hline \multicolumn{7}{|c|}{ M3 expression } \\
\hline Low & 24 & & & & 1.000 & \\
\hline High & 34 & 0.819697 & 0.356883 & 0.022 & 2.270 & I. $128-4.568$ \\
\hline
\end{tabular}

Notes: *All the $P$-values were calculated by multivariate Cox regression model. Abbreviations: $\mathrm{OS}$, overall survival; $\mathrm{SE}$, standard error; HR, hazard ratio; $\mathrm{Cl}$, confidence interval; $\mathrm{AJCC}$, American Joint Committee on Cancer; M3, muscarinic acetylcholine receptor 3 . in patient prognosis remained unknown. As is shown from the result that M3 expression is involved with lymph node invasion and AJCC stage, we predict that M3 may promote the invasion or metastasis of PDAC. The ITF has been identified as the most progressed, three to six tumor cell layers or detached tumor cell groups at the advancing edge. The ITF frequently shows a lower degree of differentiation and a higher grade of cellular dissociation in comparison with other parts of the tumor. To this end, we checked all the sections containing the ITF and found that M3 is all up-regulated in ITF/tumor budding cells; moreover, nearly half of the ITF/tumor budding cells $(11 / 29,37.9 \%)$ exhibit higher M3 expression than the central part (Figure $3 \mathrm{~A}$ and $\mathrm{B}$ ). In all the metastatic lymph nodes, M3 expression is also up-regulated $(30 / 30,100 \%)$. Compared with the central part, more than half of the metastasis cells in the lymph node exhibit higher M3 expression (17/30, 56.7\%) (Figure 3C and D).

\section{High M3 expression cells demonstrate the tendency to surround or invade into the parasympathetic nerve fibers in PDAC}

Perineural invasion is generally associated with a poor prognosis and can cause severe pain in pancreatic cancer. Recently, the presence and role of autonomic nerve fibers in the tumor microenvironment have received attention, and the infiltration of tumors by parasympathetic nerves has been evidenced and linked to cancer progression. We further investigated the patients with perineural invasion by VAChT immunochemistry and found that the tumors are all infiltrated by VAChT-positive parasympathetic nerve fibers $(26 / 26,100 \%)$. Tumor cells that surround or invade into the parasympathetic nerve fibers all show up-regulated M3 expression, and the presence of high M3 expression accounts for the majority $(22 / 26,84.6 \%)$ (Figure 4).

\section{Discussion}

First, our results demonstrated that M3 expression serves as a biomarker for the poor prognosis of PDAC. High M3 expression indicates a poor prognosis and shorter survival time in PDAC patients compared with patients with a low expression, which also related to lymph node involvement and AJCC stage. These results are in accordance with previous reports, which suggested that $\mathrm{M} 3$ is involved in the progression or prognosis in other cancers. ${ }^{5,910}$ Perineural invasion may include invasion to sympathetic nerve fiber, parasympathetic nerve fiber and sensory nerve fiber, and there is no detailed description on this 

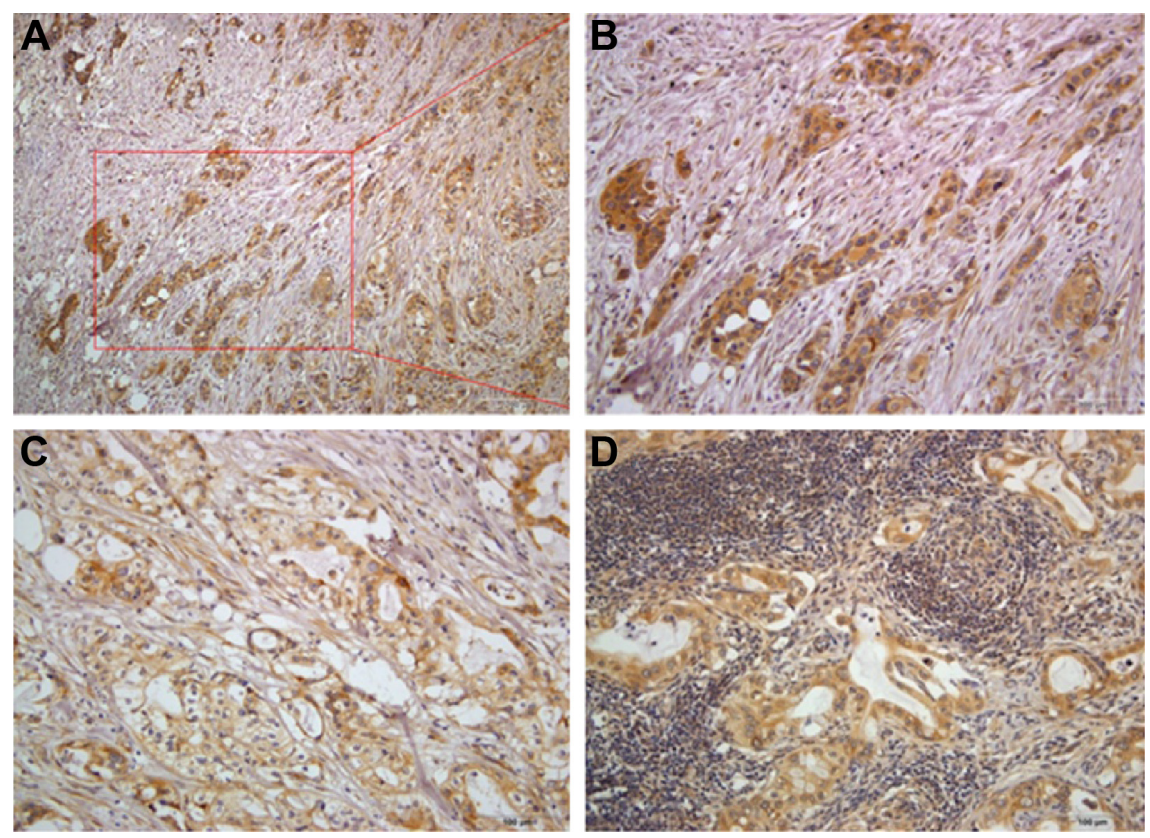

Figure 3 The unique location of high M3 expression tumor cells in ITF/tumor budding cells (A and B), tumor central part (C) and corresponding lymph node (D). Abbreviations: M3, muscarinic acetylcholine receptor 3; ITF, invasive tumor front. Magnification $(\mathbf{A}) \times 100,(\mathbf{B}-\mathbf{D}) \times 200$.
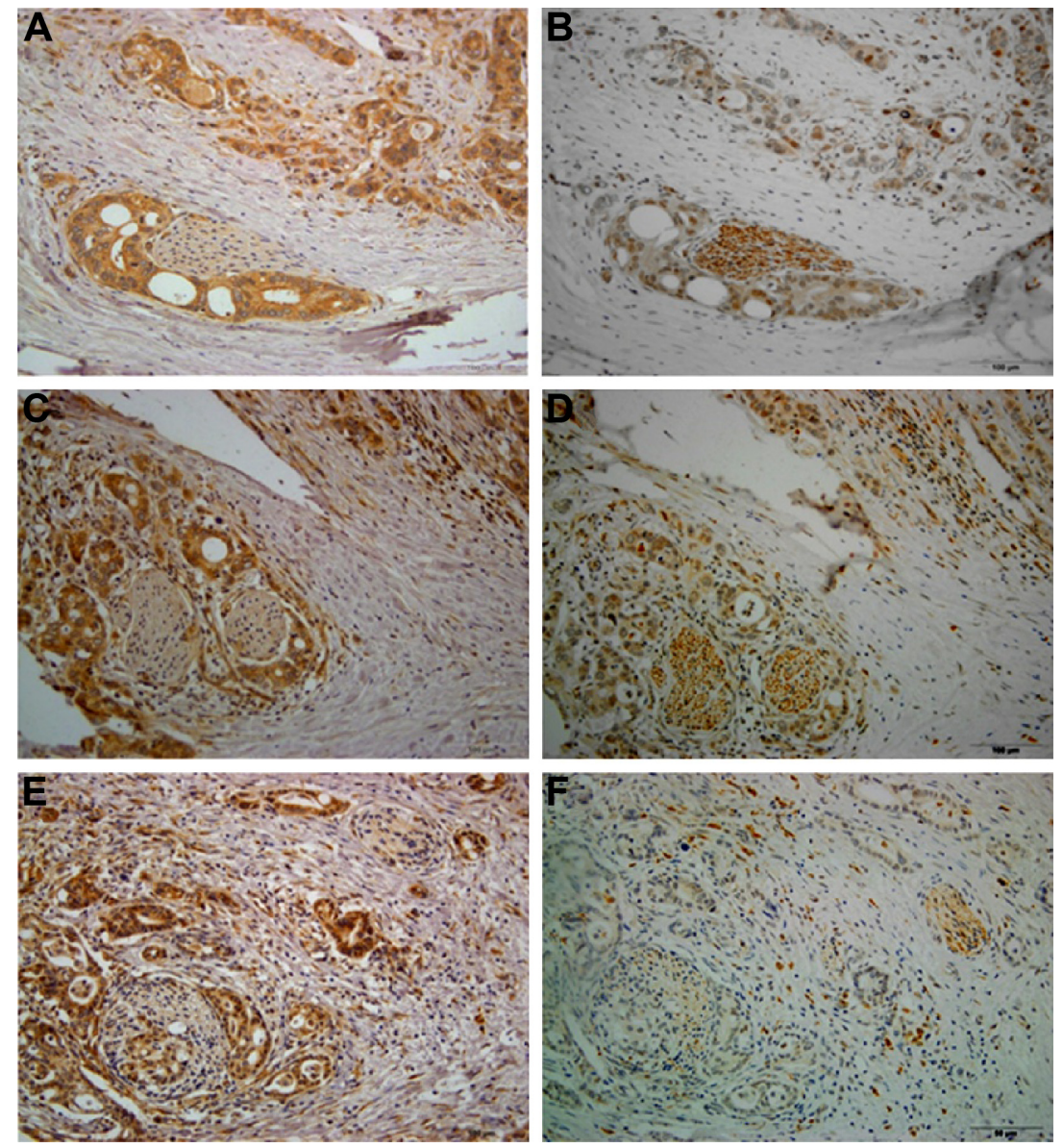

Figure 4 High M3 expression cells demonstrate the tendency to surround or invade into the parasympathetic nerve fibers in PDAC (A-C: M3 immunohistochemistry; D-F: corresponding VAChT immunohistochemistry, indicating parasympathetic nerve fibers). Magnification $\times 200$

Abbreviations: M3, muscarinic acetylcholine receptor 3; PDAC, pancreatic ductal adenocarcinoma. 
aspect till now. Although our data failed to prove the correlation between M3 expression and vessel/perineural invasion in the 58 patients, the current results demonstrated the frequent correlation between $\mathrm{M} 3$ expression and parasympathetic nerve fiber in the 26 PDAC patients who had perineural invasion. Second, M3 expression is high in ITF/tumor budding and metastatic cells in the lymph node. Numerous studies indicated that ITF and tumor budding, which reflect the invasiveness of the tumor, are indicators of poor prognosis. ${ }^{11-15}$ This study demonstrated that M3 expression is high in ITF and tumor budding cells compared with the central part of the tumor. We observed the unique high expression of M3 in a subpopulation of cancer cells at the ITF (21 of 23 cases), metastatic lymph node, but not in the central, more differentiated, part of the tumor in PDAC. This unique heterogeneity of M3 expression in PDAC has not been reported yet. Our findings suggested that M3 in PDAC could play a significant role in the invasive process in vivo. Recent investigations into the functional role of M3 in carcinomas have demonstrated that the expression of M3 augmented cell motility on extracellular matrix components ${ }^{4-7}$ and promoted tumor growth in mice ${ }^{16,17}$ in addition to its effect on cancer cell proliferation. ${ }^{18}$ These findings support our hypothesis that M3 in PDAC could play a role in the invasive process and its expression could be a more malignant phenotype. The presence of high expression of M3 was prevalent in ITF/tumor budding cells and metastatic lymph nodes, indicating a metastatic potential.

Third, high expression of M3 in tumor cells demonstrates the tendency to surround or invade into the parasympathetic nerve fibers. Perineural invasion is defined as the presence of cancer cells in the perineural space, and it is an important factor influencing the postoperative prognosis of PDAC. ${ }^{19,20}$ Although there is no statistical significance between M3 expression and perineural invasion in our result, our study shows a tendency that high-M3-expressed cells surround or invade into the VAChT-positive parasympathetic nerve fibers in patients with perineural invasion. Based on that phenomenon, we assumed that parasympathetic nerve fiber may contribute to the development of PDAC by releasing acetylcholine on cancer cells expressing M3. In fact, during perineural invasion, nerves are passive and just provide a route for cancer cell dissemination. The infiltration of tumors by growing nerves has been evidenced and linked to cancer progression only recently. ${ }^{21}$ A landmark study also demonstrated that autonomic nerve development in prostate cancer is essential to its progression. ${ }^{22}$ It took VAChT as a marker for parasympathetic nerve fiber and showed that tumors were infiltrated by parasympathetic nerve fibers, which secreted acetylcholine and promoted the cancer dissemination. Furthermore, a recent study by Zhao et $\mathrm{al}^{23}$ shows that surgical and pharmacologic denervation of the stomach strongly reduced tumor incidence and progression. Based on the above progression on parasympathetic nerve fiber and cancer metastasis, we may speculate that tumor cells with high M3 expression surrounding or invading into the parasympathetic nerve fibers reflect a reciprocal action.

The major limitation of our study is the small samples of resectable PDAC patients. However, as an initial report of the potential role of $\mathrm{M} 3$ in PDAC, our study is a well-designed prospective study that validates the use of M3 expression in the classification and treatment of patients with PDAC. These findings raise the possibility that the M3 monoclonal antibody could be applied for PDAC therapy; however, a more detailed molecular biologic mechanism of how M3 contributes to invasion and metastasis in PDAC needs to be elucidated.

\section{Conclusion}

M3 expressions were correlated with poor patient prognosis and were more frequently demonstrated at the ITF/tumor budding cells, metastatic lymph node and parasympathetic nerve fiber. Therefore, M3 expression is regarded as a novel predictor of prognosis and could be an effective target in the therapeutic strategy against PDAC.

\section{Disclosure}

This work was supported by grants from China Cancer Research Foundation Y-N2013-008 and the Doctoral Program of the Ministry of Education 20130001110089 to DX. This work was also supported by grants from the National Natural Science Foundation of China 81230051, 81472734 to HZ, the National Natural Science Foundation of China 81272709 to WF and Peking University Third Hospital grant Y81524-01 to LZ. The authors report no conflicts of interest in this work.

\section{References}

1. Bukki J. Pancreatic adenocarcinoma. N Engl J Med. 2014;371: 2139-2140.

2. Fredriksson R, Lagerstrom MC, Lundin LG, Schioth HB. The G-protein-coupled receptors in the human genome form five main families. Phylogenetic analysis, paralogon groups, and fingerprints. Mol Pharmacol. 2003;63(6):1256-1272.

3. Wess J, Eglen RM, Gautam D. Muscarinic acetylcholine receptors: mutant mice provide new insights for drug development. Nat Rev Drug Discov. 2007;6(9):721-733.

4. Kruse AC, Kobilka BK, Gautam D, Sexton PM, Christopoulos A, Wess J. Muscarinic acetylcholine receptors: novel opportunities for drug development. Nat Rev Drug Discov. 2014;13(7):549-560. 
5. Lin G, Sun L, Wang R, Guo Y, Xie C. Overexpression of muscarinic receptor 3 promotes metastasis and predicts poor prognosis in nonsmall-cell lung cancer. J Thorac Oncol. 2014;9(2):170-178.

6. Spindel ER. Muscarinic receptor agonists and antagonists: effects on cancer. Handb Exp Pharmacol. 2012;(208):451-468.

7. Von Rosenvinge EC, Raufman JP. Muscarinic receptor signaling in colon cancer. Cancers (Basel). 2011;3:971-981.

8. Zhan J, Wang P, Niu M, et al. High expression of transcriptional factor HoxB9 predicts poor prognosis in patients with lung adenocarcinoma. Histopathology. 2015;66(7):955-965.

9. Wang N, Yao M, Xu J, et al. Autocrine activation of CHRM3 promotes prostate cancer growth and castration resistance via CaM/CaMKKmediated phosphorylation of Akt. Clin Cancer Res. 2015;21(20): 4676-4685.

10. Wang Y, Li J, Wen S, et al. CHRM3 is a novel prognostic factor of poor prognosis in patients with endometrial carcinoma. Am J Transl Res. 2015;7(5):902-911.

11. Karamitopoulou E, Gloor B. Tumour budding is a strong and independent prognostic factor in pancreatic cancer. Reply to comment. Eur J Cancer. 2013;49:2458-2459.

12. Kohler I, Bronsert P, Timme S, et al. Detailed analysis of epithelialmesenchymal transition and tumor budding identifies predictors of long-term survival in pancreatic ductal adenocarcinoma. J Gastroenterol Hepatol. 2015;30(suppl 1):78-84.

13. Wartenberg M, Zlobec I, Perren A, et al. Accumulation of FOXP3+ $\mathrm{T}$ cells in the tumor microenvironment is associated with an epithelial-mesenchymal-transition-type tumor budding phenotype and is an independent prognostic factor in surgically resected pancreatic ductal adenocarcinoma. Oncotarget. 2015;6(6):4190-4201.

14. O'Connor K, Li-Chang HH, Kalloger SE, et al. Tumor budding is an independent adverse prognostic factor in pancreatic ductal adenocarcinoma. Am J Surg Pathol. 2015;39(4):472-478.
15. Karamitopoulou E, Zlobec I, Born D, et al. Tumour budding is a strong and independent prognostic factor in pancreatic cancer. Eur J Cancer. 2013;49:1032-1039.

16. Cheng K, Xie G, Khurana S, et al. Divergent effects of muscarinic receptor subtype gene ablation on murine colon tumorigenesis reveals association of M3R and zinc finger protein 277 expression in colon neoplasia. Mol Cancer. 2014;13:77.

17. Peng Z, Heath J, Drachenberg C, Raufman JP, Xie G. Cholinergic muscarinic receptor activation augments murine intestinal epithelial cell proliferation and tumorigenesis. BMC Cancer. 2013;13:204.

18. Wang L, Zhi X, Zhang Q, et al. Muscarinic receptor M3 mediates cell proliferation induced by acetylcholine and contributes to apoptosis in gastric cancer. Tumour Biol. 2016;37(2):2105-2117.

19. Demir IE, Ceyhan GO, Liebl F, D’Haese JG, Maak M, Friess H. Neural invasion in pancreatic cancer: the past, present and future. Cancers (Basel). 2010;2(3):1513-1527.

20. Takahashi T, Ishikura H, Motohara T, Okushiba S, Dohke M, Katoh H. Perineural invasion by ductal adenocarcinoma of the pancreas. J Surg Oncol. 1997;65(3):164-170.

21. Jobling P, Pundavela J, Oliveira SM, Roselli S, Walker MM, Hondermarck H. Nerve-cancer cell cross-talk: a novel promoter of tumor progression. Cancer Res. 2015;75(9):1777-1781.

22. Magnon C, Hall SJ, Lin J, et al. Autonomic nerve development contributes to prostate cancer progression. Science. 2013; 341(6142):1236361.

23. Zhao CM, Hayakawa Y, Kodama Y, et al. Denervation suppresses gastric tumorigenesis. Sci Transl Med. 2014;6(250):250ra115.
OncoTargets and Therapy

\section{Publish your work in this journal}

OncoTargets and Therapy is an international, peer-reviewed, open access journal focusing on the pathological basis of all cancers, potential targets for therapy and treatment protocols employed to improve the management of cancer patients. The journal also focuses on the impact of management programs and new therapeutic agents and protocols on

\section{Dovepress}

patient perspectives such as quality of life, adherence and satisfaction The manuscript management system is completely online and includes a very quick and fair peer-review system, which is all easy to use. Visit http://www.dovepress.com/testimonials.php to read real quotes from published authors. 